\title{
DEPOSIÇÃO ELETROASSISTIDA DE ORTOSSILICATO DE TETRAETILA (TEOS) COMO PRÉ-TRATAMENTO PARA A PINTURA DE ALUMÍNIO AAI I 00
}

\author{
Vitor Bonamigo Moreira' \\ Henrique Ribeiro Piaggio Cardoso ' \\ Jane Zoppas Ferreira' \\ Álvaro Meneguzzi '
}

\section{Resumo}

A técnica convencional de produção de filmes de organossilanos por imersão pode promover a formação de pré-tratamentos pouco homogêneos, prejudicando a sua proteção por barreira contra a corrosão e a aderência de tintas. Sendo assim, a técnica de deposição eletroassistida, baseada na promoção da eletrólise da água e consequente favorecimento das reações de condensação do organossilano, é estudada com o objetivo de promover a formação de filmes com menos descontinuidades. Neste trabalho a deposição eletroassistida foi comparada com a técnica convencional de imersão, bem como com a cromatização hexavalente. O precursor utilizado neste trabalho foi o ortossilicato de tetraetila (TEOS), um silano não funcionalizado. A comparação entre os pré-tratamentos foi realizada através de ensaios de corrosão acelerada em névoa salina, medida de aderência de tinta, espectroscopia de impedância eletroquímica e espectroscopia de energia dispersiva. Os resultados indicam que a deposição eletroassistida em potenciais catódicos promoveu maior cobertura do substrato.

Palavras-chave: Silano; Deposição eletroassistida; Pré-tratamento; Alumínio.

\section{TETRAETHYL ORTHOSILICATE (TEOS) ELECTRO-ASSISTED DEPOSITION AS A PRETREATMENT FOR PAINTING AAIIOO ALUMINUM ALLOY}

\begin{abstract}
The standard method for the production of organosilane films by dip-coating may lead to non-homogeneous pretreatment layers, weakening its barrier protection against corrosion and paint adhesion. Therefore, the electro-assisted deposition technique, based on promoting water electrolysis and the consequent favoring of condensation reactions of the organosilane, is studied with the aim of promoting the formation of less discontinuous coatings. In this work the electro-assisted deposition was compared to the standard dip-coating method and to hexavalent chromate conversion coating. The precursor used in this work was tetraethyl orthosilicate (TEOS), a non-functional silane. The comparison between the pretreatments was carried out by salt spray tests, paint adhesion measurement, electrochemical impedance spectroscopy and energy dispersive spectroscopy. The results indicate that the electro-assisted deposition in cathodic potentials promoted higher coverage of the substrate.
\end{abstract}

Keywords: Silane; Electro-assisted deposition; Pretreatment; Aluminum.

\section{INTRODUÇÃO}

A proteção por barreira fornecida pela formação natural da camada de óxido de alumínio resulta em aumento da resistência contra a corrosão [ 1$]$, porém, há casos onde existe a necessidade de proteção adicional, com o uso de revestimentos orgânicos. Esta barreira de óxido pode representar um inconveniente, uma vez que a sua inércia química prejudica a aderência de tais revestimentos, tornando necessário o uso de pré-tratamentos [2]. Dentre estes

'Laboratório de Corrosão, Proteção e Reciclagem de Materiais, Universidade Federal do Rio Grande do Sul - UFRGS, Porto Alegre, RS, Brasil. E-mail: vitor.bonamigo@hotmail.com 
pré-tratamentos destaca-se a cromatização hexavalente, que promove a aderência de tintas e fornece proteção satisfatória contra a corrosão abaixo do filme orgânico [3].

Apesar do bom desempenho em diversos ambientes e substratos, o uso da cromatização, aceito por várias décadas, encontra atualmente resistência em função do seu potencial poluidor. $O$ processo gera efluentes ricos em cromo hexavalente que representam risco em caso de disposição e tratamento inadequados [4]. Mesmo que existam estudos visando o tratamento mais eficiente de poluentes contendo cromo [5], há um esforço global na busca por alternativas ambientalmente mais amigáveis que a cromatização [6], como revestimentos nanocerâmicos [7], revestimentos de conversão à base de terras raras [8], molibdato [9], manganato [10] e revestimentos à base de organossilanos [I I], objeto de estudo do presente trabalho.

Os organossilanos encontraram sua primeira aplicação em larga escala como agentes de acoplamento em compósitos poliméricos reforçados por fibras de vidro, em virtude do seu caráter híbrido orgânico/inorgânico, o que permite que esses apresentem afinidade química tanto com a fibra inorgânica quanto com a matriz orgânica [I2]. Valendo-se desta mesma característica, os organossilanos podem ser utilizados para a produção de revestimentos como pré-tratamento para a pintura do alumínio [13], uma vez que a escolha de um organossilano com um grupamento funcional com afinidade química com o revestimento orgânico pode resultar em ligação química entre o pré-tratamento e a tinta [14].

O método de imersão simples em sol-gel, usualmente empregado para a confecção de filmes de organossilanos, pode proporcionar a formação de filmes pouco homogêneos e com propriedades de barreira prejudicadas em função da sua alta dependência da preparação prévia e das características superficiais do substrato $[\mathrm{II}, 15]$.

A técnica de deposição eletroassistida de organossilanos, alternativa à imersão simples, pode conduzir à formação de filmes mais homogêneos, com melhores propriedades de barreira [16]. A técnica baseia-se na promoção da eletrólise da água da solução de imersão, cuja reação catódica produz hidroxilas, ocasionando um aumento localizado do $\mathrm{pH}$ no cátodo (peça a tratar), favorecendo a ocorrência das reações de condensação dos organossilanos hidrolisados [17].

O presente trabalho tem como objetivo a comparação de filmes produzidos pelo processo de deposição eletroassistida de TEOS com filmes oriundos da técnica convencional de imersão, verificando-se a eficiência da técnica como pré-tratamento para a pintura de alumínio. Adicionalmente, estes filmes foram comparados com revestimentos de conversão à base de cromo hexavalente e com alumínio sem pré-tratamento. Neste trabalho foi utilizado um ortossilicato de baixo custo e sem grupamento funcional. Nesse contexto, o pré-tratamento não deverá ter afinidade química com o revestimento, logo a formação bem-sucedida de filmes poderá resultar em diminuição da aderência do revestimento orgânico. A comparação foi realizada através de análises de corrosão acelerada em névoa salina, medida da aderência de revestimento orgânico por tração, mapeamento de silício por espectroscopia de energia dispersiva (EDS) e espectroscopia de impedância eletroquímica (EIE).

\section{MATERIAIS E MÉTODOS}

Nesta seção serão apresentados os materiais utilizados neste trabalho, bem como a metodologia de produção e análise dos revestimentos estudados.

\section{I Preparo das Amostras}

O substrato metálico utilizado neste trabalho foi o alumínio comercialmente puro AA I I 00, em placas de dimensões $100 \times 50 \times 0,5 \mathrm{~mm}$. Estas peças foram desengraxadas com pano embebido em acetona (Acetona P.A. - Neon Comercial Ltda.), seguido por imersão em desengraxante alcalino (Saloclean 667N - Klintex Insumos Industriais Ltda.) a $70^{\circ} \mathrm{C}$ durante 5 minutos. Após a imersão em desengraxante, as placas foram enxaguadas com água destilada deionizada, sendo posteriormente secas com jato de ar quente.

A solução utilizada para a produção dos revestimentos por sol-gel foi composta, em proporções volumétricas, por $4 \%$ de TEOS (Aldrich Chemistry 98\%), 48\% de álcool etílico (Synth ${ }^{\circledR} 95 \%$ P. A.) e $48 \%$ de água destilada. $\mathrm{O}$ pH da solução foi regulado a 2,5 com a adição de ácido acético glacial $\left(\right.$ Synth $\left.^{\circledR}\right)$. Após o preparo e regulagem do $\mathrm{pH}$, a solução foi mantida sob agitação por 24 horas para a ocorrência das reações de hidrólise do TEOS.

A produção dos revestimentos de TEOS por imersão foi realizada em um Elevador de Discos MA 765 (Marconi Equipamentos para Laboratórios Ltda.), com tempo de imersão de 5 minutos e retirada à taxa de $210 \mathrm{~mm} / \mathrm{min}$. Para as amostras produzidas por deposição eletroassistida foi utilizada uma célula de três eletrodos, sendo um eletrodo de referência de $\mathrm{Ag} \mid \mathrm{AgCl}$, contra eletrodos de aço inoxidável AISI 316 e a placa a ser revestida como o eletrodo de trabalho, ligada a um potenciostato (Autolab PGSTAT 302, software de operação Nova I.II). Após a montagem das células, foi medido o potencial de circuito aberto (OCP) dos sistemas durante 10 segundos. Findada esta medição, o sobrepotencial de deposição foi aplicado às células, com relação aos seus respectivos valores de OCP.

Tanto as amostras revestidas por imersão quanto por deposição eletroassistida passaram por secagem em estufa a $50^{\circ} \mathrm{C}$ por 10 minutos para a remoção do excesso de líquidos presente nos filmes, após a qual a cura dos revestimentos de TEOS foi realizada durante 60 minutos a $125^{\circ} \mathrm{C}$.

As peças cromatizadas passaram do desengraxe à imersão em solução cromatizante hexavalente (Cromatizante Hexavalente 674B - SurTec do Brasil Ltda.) por 5 minutos, seguida de retirada à taxa de $210 \mathrm{~mm} / \mathrm{min}$, com o equipamento elevador de discos anteriormente citado. Este procedimento foi sucedido com secagem por jato de ar quente.

As amostras sem pré-tratamento seguiram da etapa de desengraxe diretamente à etapa de pintura.

A aplicação do revestimento orgânico (Verniz alquídico base solvente - Extra Esmalte - Tintas Renner) foi realizada para todas as amostras por imersão em equipamento Elevador de Discos MA 765 durante 5 segundos, seguida de retirada 
a $210 \mathrm{~mm} / \mathrm{min}$. Após a pintura, as amostras passaram por um período de cura por exposição ao ar atmosférico à temperatura ambiente por 7 dias.

Ao término deste procedimento as amostras receberam as seguintes denominações: Branco, para as amostras sem pré-tratamento; Cromatizado, para amostras cromatizadas; Ime para as amostras que passaram somente pela deposição via dip-coating; $+0,4 \mathrm{~V},-0,2 \vee$ e $-0,6 \vee$ para as amostras produzidas por deposição eletroassistida nesses respectivos sobrepotenciais.

\subsection{Ensaios}

Tendo em vista a verificação das faixas de potenciais associadas a correntes catódicas e anódicas para o sistema de deposição eletroassistida, bem como a ocorrência, ou não, de reações de oxidação/redução quando da aplicação das diferenças de potencial empregadas no procedimento de deposição eletroassistida, foi realizada em potenciostato (Autolab PGSTAT 302, software de operação Nova I.I I) a voltametria nas células de deposição eletroassistida reproduzindo a montagem utilizada para a deposição, com - eletrodo de trabalho de alumínio, contra eletrodos de aço inoxidável, um eletrodo de referência de $\mathrm{Ag} \mid \mathrm{AgCl}$ e a solução do precursor como eletrólito. A varredura de potenciais foi conduzida de de $-1,5 \mathrm{Va}+1,0 \mathrm{~V}$ com relação ao $\mathrm{OCP}$, à taxa de $0,0 \mathrm{I} \mathrm{V/s}$. Este ensaio foi realizado em triplicata, sendo uma destas repetições apresentada na Figura $I$.

A análise de EDS (Zeiss EVO MAI0) foi conduzida para mapear-se o silício, indicador da presença do filme de organossilano, presente na superfície. Para tanto, foi realizada a análise por área a uma magnificação de $52 x$ de uma amostra de cada pré-tratamento. $O$ resultado desta análise são imagens de fundo preto, mostradas na Figura 3, onde são marcados pontos em branco e em tons de cinza referentes à presença de silício. Com o uso de um software de análise de imagem (Image) foi calculada a área proporcional contendo silício nestes mapas.

O ensaio de corrosão acelerada em névoa salina foi conduzido conforme a norma ABNT NBR 8094, com avaliação do grau de corrosão a cada 24 horas. Os graus de corrosão vão de 0 (zero) a 5 , sendo 0 a ausência de corrosão e 5 a corrosão generalizada da peça. A exemplo do ensaio de voltametria, este ensaio foi realizado em triplicata para cada um dos revestimentos. Para a comparação entre os diferentes pré-tratamentos (Figura 2 ) foi selecionada uma amostra representativa de cada um destes.

Os ensaios de EIE foram realizados utilizando-se - potenciostato mencionado anteriormente com uma célula de três eletrodos, com um eletrodo de referência de $\mathrm{Ag} \mid \mathrm{AgCl}$, contra eletrodo de platina e a amostra a ser analisada como eletrodo de trabalho, com área de ensaio de $0,64 \mathrm{~cm}^{2}$. O eletrólito utilizado foi uma solução aquosa de $\mathrm{NaCl} 0$, I M. No ensaio de EIE, foi realizada a medida da impedância a partir de uma excitação de amplitude de $10 \mathrm{mV}_{\text {rms }}$ em relação ao OCP de cada célula, medido previamente ao longo de 60 segundos, em frequências de $10^{6} \mathrm{~Hz}$ a $10^{-1} \mathrm{~Hz}$. As medidas de EIE foram realizadas 24 horas após a montagem das células, isto é, após imersão das áreas ensaiadas no eletrólito. Visando minimizar a influência de eventuais heterogeneidades nos resultados, este ensaio foi realizado com cinco repetições em cinco amostras diferentes para cada revestimento, sendo que os diagramas de Nyquist apresentados na Figura 4 são correspondentes a amostras selecionadas de modo a expor representativamente o grupo de cinco amostras.

A medida de aderência do revestimento orgânico com equipamento portátil (DeFelsko PosiTest AT-A) foi realizada de acordo com a norma ASTM D454 I-09. Foram empregadas dollies de $20 \mathrm{~mm}$ de diâmetro e a taxa de tração utilizada foi de $0,2 \mathrm{MPa} / \mathrm{s}$ para a medida em cinco amostras para cada sistema de revestimento.

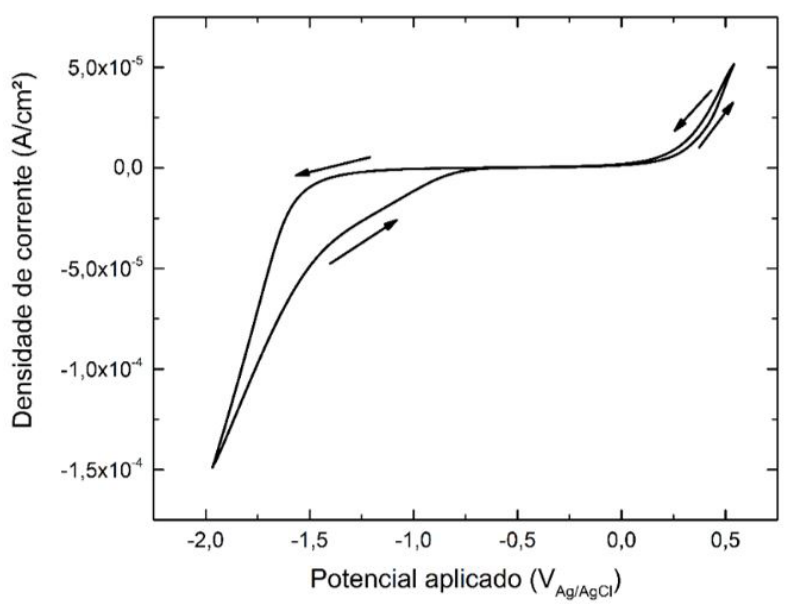

Figura I. Voltamograma conduzido na célula de deposição eletroassistida com substrato de alumínio AA I I 00 em sol-gel de TEOS a partir da varredura de potenciais entre $-I, 5 \vee$ e $+I, 0 \mathrm{~V}$ a partir do OCP.

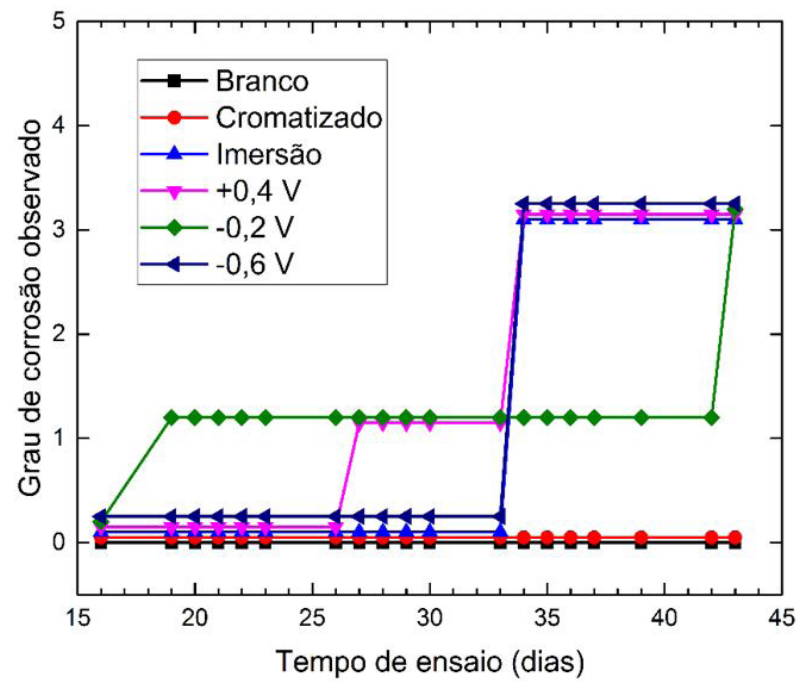

Figura 2. Representação gráfica dos graus de corrosão observados ao longo do ensaio de corrosão acelerada em névoa salina. 

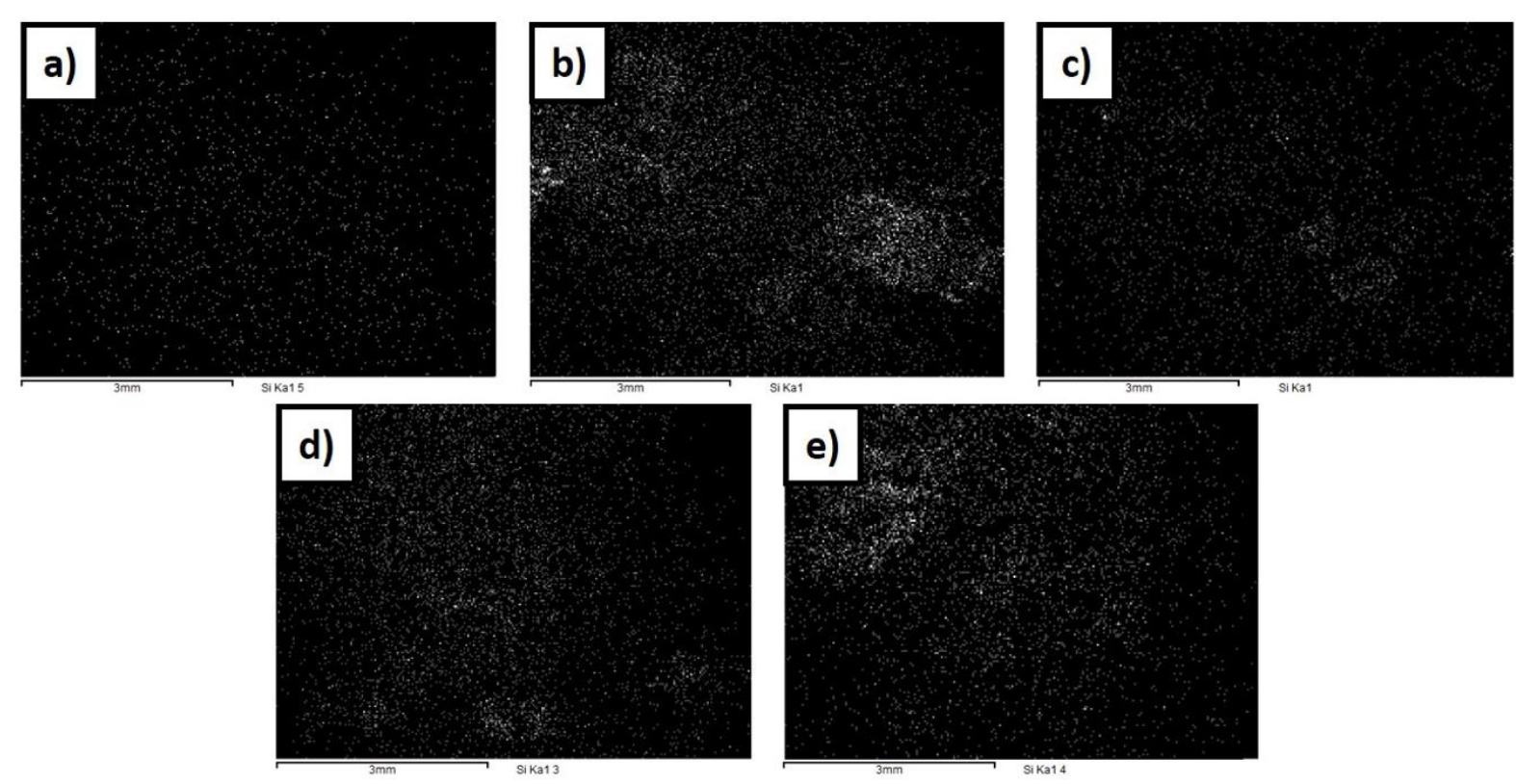

Figura 3. Mapas de silício obtidos por EDS de amostras revestidas com TEOS. (a) Branco, (b) Imersão, (c) + 0,4 V, (d) - 0,2 V e (e) - 0,6 V.

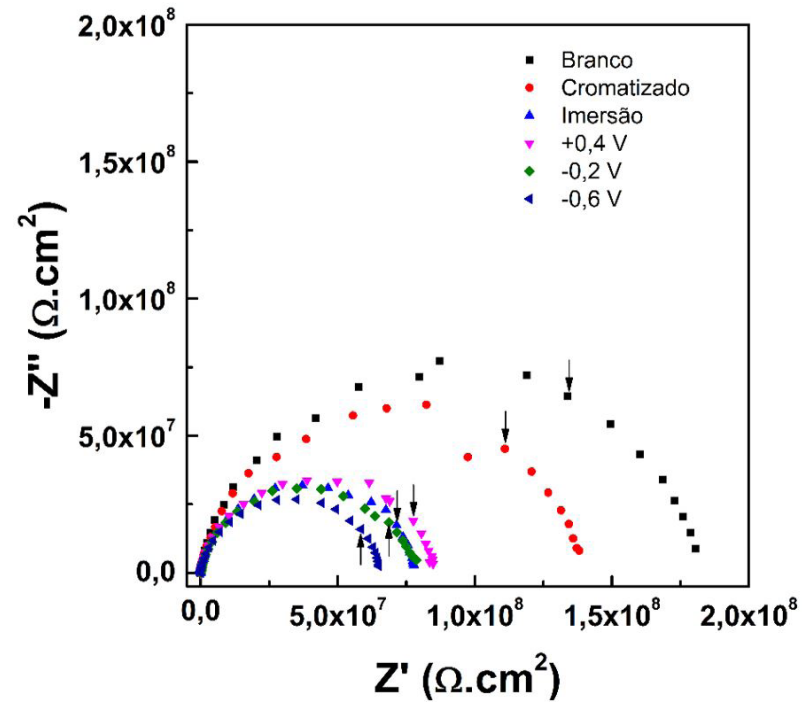

Figura 4. Espectroscopia de impedância eletroquímica em diagrama de Nyquist para 24 horas de imersão em $0,1 \mathrm{M}$ de $\mathrm{NaCl}$ de corpos-de-prova de alumínio AAII00 com os pré-tratamentos estudados. As setas indicam os pontos referentes à frequência de excitação de $\mathrm{I} \mathrm{Hz}$.

\section{RESULTADOS E DISCUSSÃO}

Nesta seção serão apresentados os resultados observados nos ensaios descritos na seção 2.3 .

\section{I Voltametria}

O ensaio de voltametria realizado na célula de deposição eletroassistida simulando a montagem utilizada para as deposições de TEOS resultou no voltamograma mostrado na Figura I.
O OCP medido para a célula foi de $-0,45 \mathrm{~V}_{\mathrm{Ag} \mid \mathrm{AgCl}}$ $\mathrm{Na}$ faixa de potenciais próximas ao OCP medido, as densidades de corrente observadas são relativamente baixas, dando um indicativo de que sobrepotenciais próximos deste valor podem não auxiliar significativamente a formação do filme de TEOS.

Não é evidente a ocorrência de nenhuma reação de redução ou oxidação das espécies presentes na solução, logo é bastante provável que a única reação que ocorre durante o procedimento seja a eletrólise da água.

A partir destas observações, foram selecionados os seguintes sobrepotenciais para a deposição eletroassistida: $+0,4 \mathrm{~V}$, potencial anódico, para verificar a validade da teoria na qual se baseia a técnica, segundo a qual a formação dos filmes é favorecida apenas em sobrepotenciais catódicos; - $0,2 \mathrm{~V}$, com o objetivo de avaliar o uso de um potencial catódico correspondente a uma baixa densidade de corrente; $-0,6 \mathrm{~V}$, tendo em vista a obtenção bem-sucedida de filmes de TEOS a partir da deposição eletroassistida em potencial catódico.

\subsection{Névoa Salina}

Os graus de corrosão observados ao longo do ensaio de névoa salina são representados graficamente na Figura 2. Vale destacar que os graus de corrosão não possuem dígito decimal, portanto, para evitar a sobreposição de séries foi acrescentado a cada uma delas um dígito decimal diferente. Assim sendo, todos os pontos localizados entre dois graus de corrosão são, na verdade, referentes ao mais baixo destes dois.

As amostras Branco e Cromatizado apresentaram a maior resistência à corrosão, não tendo apresentado nenhum ponto de corrosão ao longo dos 43 dias do ensaio. Já as amostras com os pré-tratamentos de TEOS 
apresentaram grau 3 de corrosão ao término do ensaio, indicando que os filmes formados prejudicam a proteção contra a corrosão, possivelmente em função da degradação do filme de óxido de alumínio natural ao longo da formação dos filmes. Esta degradação pode ser decorrente do contato com a solução de TEOS, cujo $\mathrm{pH}$ se encontra na região de corrosão, no diagrama de Pourbaix do alumínio [I].

Adicionalmente, não há consenso a respeito da proteção fornecida por filmes de TEOS, uma vez que há autores que reportam a obtenção de revestimentos protetores de TEOS [18], enquanto outros não detectam melhoria significativa na proteção contra a corrosão quando do uso do mesmo [19]. Sabe-se que o TEOS é utilizado para auxiliar a formação de filmes de outras moléculas, uma vez que ele facilita a reticulação do filme por possuir quatro grupos hidrolisáveis e nenhum grupamento funcional que forneceria propriedades de aderência do revestimento orgânico ou efeito repelente ao eletrólito [20]. Além disso, sabe-se que a reação de hidrólise dos organossilanos é reversível, portanto a presença de grupamentos hidrolisados não condensados possibilita a diminuição da proteção fornecida pelo filme [I I].

\subsection{Aderência de Revestimento Orgânico}

Os valores de aderência do revestimento orgânico medidos por tração são mostrados na Tabela I.

Houve uma influência negativa do uso do TEOS na aderência do revestimento orgânico, sendo que os maiores valores foram observados para as amostras Branco e Cromatizado. Nota-se também que o uso do sobrepotencial anódico $(+0,4 \mathrm{~V})$ para a deposição eletroassistida ocasionou uma diminuição menor da aderência em comparação aos potenciais catódicos e à imersão.

A ausência de grupamentos organofuncionais na molécula de TEOS explica a diminuição da aderência quando do seu uso, uma vez que não há no filme organometálico formado grupamentos com afinidade química com o verniz.

O fato de o sobrepotencial anódico resultar em uma diminuição menor da aderência em comparação às demais amostras revestidas com TEOS dá indício de que para este sobrepotencial não há formação bem-sucedida de um filme de TEOS, uma vez que a presença do TEOS se mostrou prejudicial à aderência.

\subsection{EDS}

As imagens da distribuição de silício, cuja presença é representada pelos pontos brancos e em tons de cinza, obtidas por EDS são mostradas na Figura 3.

Os mapas de silício indicam que o sobrepotencial anódico (Figura 3c) pouco alterou a presença de silício em comparação à amostra sem pré-tratamento (Figura 3a), que pode ser considerada como o ruído de fundo da análise. Nota-se a distribuição pouco homogênea decorrente do processo de imersão (Figura 3b).
Estas observações vão ao encontro das observações feitas por outros autores, que observaram menor homogeneidade em filmes produzidos por imersão quando comparados à deposição eletroassistida [16], [2I], e confirmam a ineficiência do uso de potenciais anódicos no processo.

A partir das imagens da Figura 3 foram calculadas as áreas percentuais onde é detectado o silício para cada pré-tratamento. Tais resultados são apresentados na Tabela 2.

Corroborando a observação anterior, o sobrepotencial $+0,4 \bigvee$ não proporcionou uma formação bem-sucedida do filme de TEOS. A amostra -0,6 V apresentou a maior proporção de área contendo silício, indicando uma distribuição mais homogênea do filme. $O$ sobrepotencial -0,2 $\vee$ ocasionou menor presença de silício que a imersão, dando indício de que baixas densidades de corrente podem não auxiliar significativamente a formação do filme.

Apesar de o uso de sobrepotenciais catódicos ter resultado em melhor cobertura que o sobrepotencial anódico, é possível que maior homogeneidade possa ser obtida a partir do uso de sobrepotenciais diferentes, como o sobrepotencial -0,8 V, indicado por outros autores [2I-23] como o sobrepotencial mais adequado para o uso da técnica.

\subsection{Espectroscopia de Impedância Eletroquímica}

A partir dos resultados da espectroscopia de impedância eletroquímica obtidos nas amostras submersas em $0,1 \mathrm{M}$ de $\mathrm{NaCl}$ por 24 horas foram criados os diagramas de Nyquist que estão representados na Figura 4.

Pode-se tirar conclusões com base no tamanho e formato do arco formado no diagrama de Nyquist. Deste modo, observa-se que o melhor desempenho foi alcançado pelo

Tabela I. Medidas de aderência de revestimento orgânico por tração com equipamento portátil para todos os pré-tratamentos estudados

\begin{tabular}{ccc}
\hline Pré-tratamento & $\begin{array}{c}\text { Aderência média } \\
(\mathbf{M P a})\end{array}$ & $\begin{array}{c}\text { Desvio padrão } \\
\mathbf{( M P a )}\end{array}$ \\
\hline Branco & 1,40 & 0,16 \\
Cromatizado & 1,42 & 0,13 \\
Imersão & 0,85 & 0,16 \\
$+0,4 \mathrm{~V}$ & 1,05 & 0,01 \\
$-0,2 \mathrm{~V}$ & 0,70 & 0,11 \\
$-0,6 \mathrm{~V}$ & 0,82 & 0,18 \\
\hline
\end{tabular}

Tabela 2. Áreas proporcionais contendo silício calculadas a partir dos mapas de silício obtidos por EDS

\begin{tabular}{cc}
\hline Pré-tratamento & $\begin{array}{c}\text { Área proporcional } \\
\text { contendo silício (\%) }\end{array}$ \\
\hline Branco & 3,3 \\
Imersão & 14,7 \\
$+0,4 \mathrm{~V}$ & 6,2 \\
$-0,2 \mathrm{~V}$ & 10,1 \\
$-0,6 \mathrm{~V}$ & 17,1 \\
\hline
\end{tabular}


sistema sem revestimento, corroborando as observações realizadas nos demais experimentos. A amostra cromatizada apresentou o segundo melhor resultado, possivelmente pelo fato de o tempo de contato com o eletrólito não ser o suficiente para que os íons cromato solúveis atuem na passivação do substrato. Já os revestimentos de silano apresentaram resultados semelhantes, todos abaixo das amostras Branco e Cromatizado, fato possivelmente explicado pela falta de afinidade química entre a tinta e o TEOS e pela degradação da camada natural de óxido de alumínio pela imersão em pH 2,5.

\section{CONCLUSÃO}

O uso da técnica de deposição eletroassistida não ocasiona a ocorrência de reações de redução ou oxidação nos componentes da solução de tratamento estudada.

A proteção contra a corrosão fornecida pelos filmes de TEOS mostrou-se ineficiente, possivelmente em decorrência de dois fatores: a falta de afinidade química entre o silano e 0 revestimento orgânico, bem como a degradação da camada de óxido natural de alumínio em função da imersão em uma solução com pH desfavorável a esse metal.

Observou-se que a presença do TEOS resultou em diminuição da aderência de tinta, e o uso de um sobrepotencial anódico na deposição resulta em menor perda de aderência, fruto de uma formação malsucedida do filme.

O uso da técnica de deposição eletroassistida em sobrepotenciais catódicos ocasionou a formação de filmes com distribuição mais homogênea de silício quando comparados àqueles produzidos por imersão, mostrando-se uma alternativa promissora para o uso de um silano organofuncional, cuja afinidade química com o revestimento orgânico promoveria melhora na aderência deste.

\section{Agradecimentos}

Os autores agradecem à equipe do Laboratório de Corrosão, Proteção e Reciclagem de Materiais (LACOR) da Universidade Federal do Rio Grande do Sul e ao Conselho Nacional de Desenvolvimento Científico e Tecnológico (CNPq).

\section{REFERÊNCIAS}

I Vargel C. Corrosion of aluminium. Amsterdam: Elsevier; 2004.

2 Joseph R. ABCs of painting on aluminum. Metal Finishing. 2008;6(2):39-44.

3 Ita AO. Paint pretreatments for aluminum. Metal Finishing. 1999;97(5):87-91.

4 Xue D, Van Ooij WJ. Corrosion performance improvement of hot-dipped galvanized (HDG) steels by electrodeposition of epoxy-resin-ester modified bis-[tri-ethoxy-silyl] ethane (BTSE) coatings. Progress in Organic Coatings. 2013;76(7-8): 1095-1102.

5 Kalidhasan S, Santhana Krishna Kumar A, Rajesh V, Rajesh N. The journey traversed in the remediation of hexavalent chromium and the road ahead toward greener alternatives-a perspective. Coordination Chemistry Reviews. 2016;317:157-166.

6 Fedel M. Environmentally friendly hybrid coatings for corrosion protection: silane based pre-treatments and nanostructured waterborne coatings. Trento: University of Trento; 2009.

7 Costa JS, Agnoli RD, Ferreira JZ. Corrosion behavior of a conversion coating based on zirconium and colorants on galvanized steel by electrodeposition. Tecnologica em Metalurgia, Materiais e Mineração. 20I5; 12(2): 167- 175.

8 Pommiers S, Frayret J, Castetbon A, Potin-Gautier M. Alternative conversion coatings to chromate for the protection of magnesium alloys. Corrosion Science. 2014;84:135-I46.

9 Zhang M, Mu S, Guan Q, Li W, Du J. A high anticorrosive chromium-free conversion coating prepared with an alkaline conversion bath on electroless Ni-P coating. Applied Surface Science. 20I5;349: I08-I I 5.

10 Gan Y, Chen D, Kang Z. A highly anticorrosive chromium-free conversion coating prepared on electroless Ni-P coating. Surface and Coatings Technology. 2016;287:25-32.

I I Van Ooij WJ, Zhua D, Stacy M, Seth A, Mugada T, Gandhia P, et al. Corrosion protection properties of organofunctional silanes_-an overview. Tsinghua Science and Technology. 2005;10(6):639-664.

12 Plueddemann EP. Principles of interfacial coupling in fibre-reinforced plastics. International Journal of Adhesion and Adhesives. 198I; I (6):305-310.

13 Iribarren-Mateos JI, Buj-Corral I, Vivancos-Calvet J, Alemán C, Iribarren JI, Armelin E. Silane and epoxy coatings: a bilayer system to protect AA2024 alloy. Progress in Organic Coatings. 2015;81:47-57. 
I4 Zheludkevich ML, Salvado IM, Ferreira MGS. Sol-gel coatings for corrosion protection of metals. Journal of Materials Chemistry. 2005;I5(48):5099.

I5 Díaz-Benito B, Velasco F, Pantoja M. Mechanical properties of polyester films painted after silanization of 6063 aluminium alloy with different pretreatment conditions. Progress in Organic Coatings. 201 I;70(4):287-292.

16 Gandhi JS. Electrodeposition of organofunctional silanes for improved corrosion protection of metals. Cincinnati: University of Cincinnati; 2004.

17 Shacham R, Avnir D, Mandler D. Electrodeposition of methylated sol-gel films on conducting surfaces. Advanced Materials. 1999; I I(5):384-388.

18 Hashem KME. Study of TEOS and TPOS anticorrosion coatings developed at different ranges of pyrolysis temperatures. Applied Surface Science. 2003;217( I-4):302-313.

19 Sakai RT, Cruz FMDL, Melo HG, Benedetti AV, Santilli CV, Suegama PH. Electrochemical study of TEOS, TEOS/ MPTS, MPTS/MMA and TEOS/MPTS/MMA films on tin coated steel in $3.5 \% \mathrm{NaCl}$ solution. Progress in Organic Coatings. 20I2;74(2):288-30I.

20 Yan H, Yuanhao W, Hongxing Y. TEOS/silane coupling agent composed double layers structure: A novel superhydrophilic coating with controllable water contact angle value. Applied Energy. 2015

2I Hu J-M, Liu L, Zhang J-Q, Cao C-N. Effects of electrodeposition potential on the corrosion properties of bis-1,2[triethoxysilyl] ethane films on aluminum alloy. Electrochimica Acta. 2006;5 I (I9):3944-3949.

$22 \mathrm{Hu}$ J-M, Liu L, Zhang J-Q, Cao C-N. Electrodeposition of silane films on aluminum alloys for corrosion protection. Progress in Organic Coatings. 2007;58(4):265-27I.

23 Li M, Yang Y-Q, Liu L, Hu J-M, Zhang J-Q. Electro-assisted preparation of dodecyltrimethoxysilane/TiO2 composite films for corrosion protection of AA2024-T3 (aluminum alloy). Electrochimica Acta. 20 I0;55(8):3008-30 I4.

Recebido em: 10 Jan. 2017

Aceito em: 26 Abr. 2017 